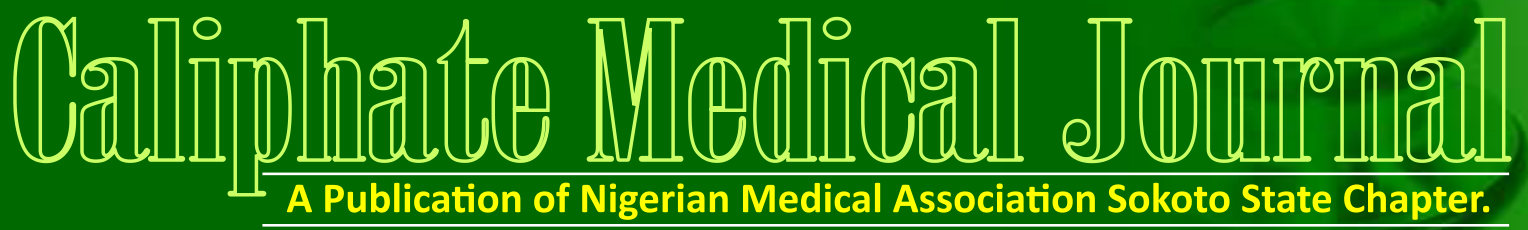

Vol 9, No. 2, April - June, 2021

ISSN: 2346-7193

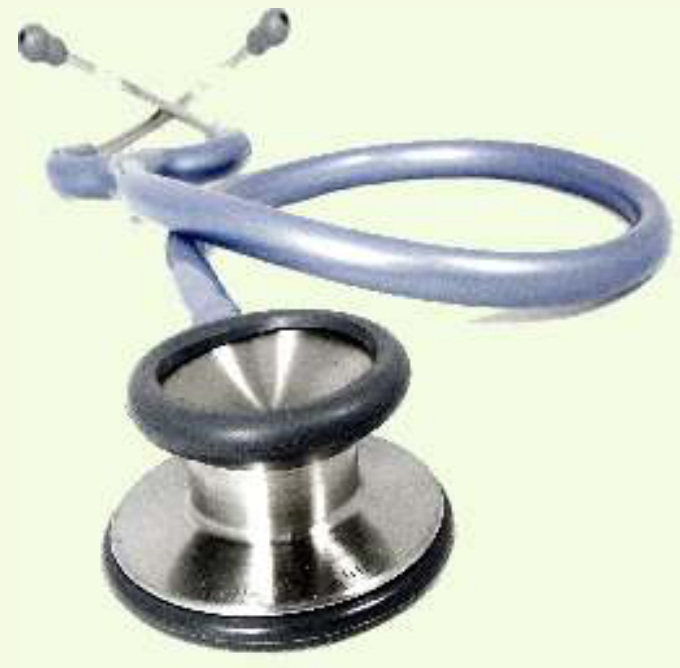

In this Issue

O

O

O

O

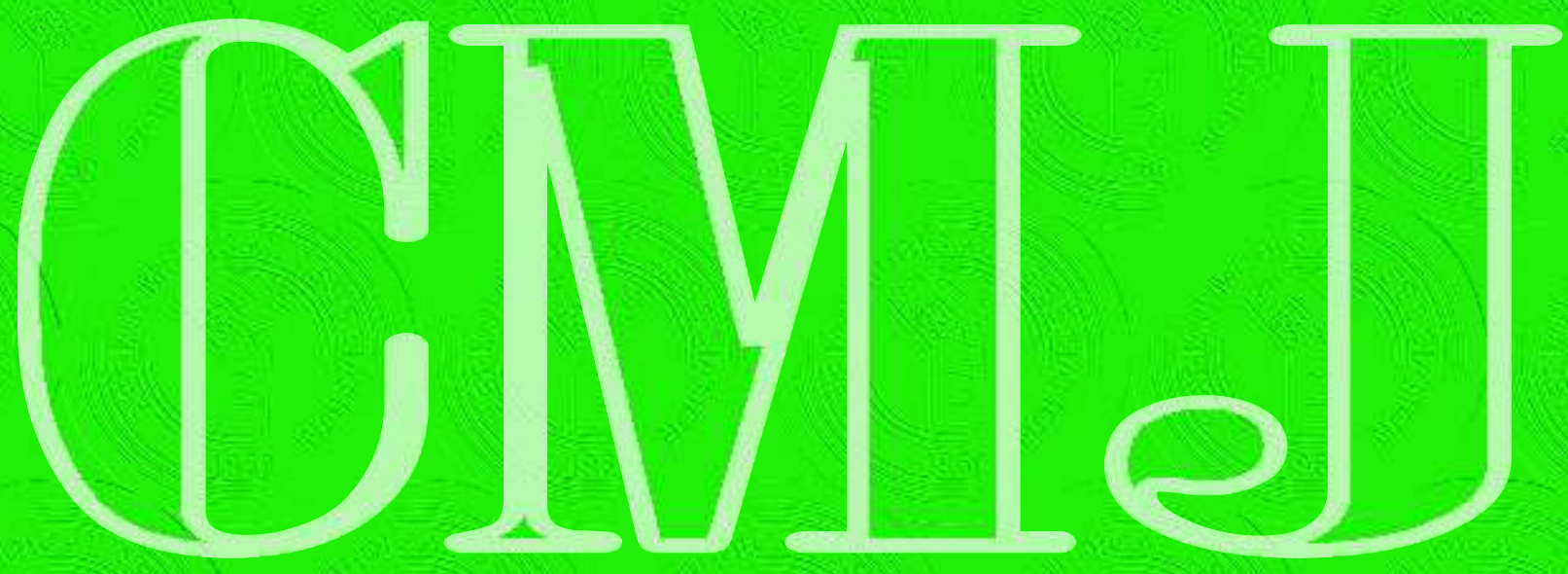




\section{OSTEOSARCOMA OF THE SKULL: A RARE CLINICAL} CONDITION

Lasseini, A, Aliyu, MK, Nasiru, JI, Shehu, BB

Department of Neurosurgery, Usmanu Danfodiyo University Teaching Hospital Sokoto, Nigeria.

\begin{tabular}{r|r}
\hline Corresponding author: & ABSTRACT \\
Ali Lasseini, & $\begin{array}{r}\text { Introduction: Osteosarcoma is an uncommon malignant bone tumour that may rarely involve the skull bones. } \\
\text { We report two cases of skull tumours that had surgical excision and histopathology confirmed osteosarcoma. }\end{array}$ \\
$\begin{array}{r}\text { Department of Neurosurgery, } \\
\text { Usmanu Danfodiyo University Teaching Hoppital } \\
\text { Sokoto, Nigeria. }\end{array}$ & $\begin{aligned} \text { Keywords: Osteosarcoma, skull tumour, surgical excision } \\
\text { Email: lassuduth@gmail.com }\end{aligned}$
\end{tabular}

\section{Introduction}

$\mathbf{O}$ steosarcoma of the skull is an extremely rare neoplastic condition encountered in neurosurgical practice. Osteosarcomas are described as spindle-cell tumors with abundant irregular and immature bones. ${ }^{[1]}$ It commonly involves long bone and accounts for 1-2\% of all skull tumours. ${ }^{[2-4]}$ Skull osteosarcoma represents about $6-8 \%$ of all osteosarcomas and occurs mostly in third to fourth decades of life ${ }^{[4]}$ Is of note that, fewer than 150 cases have been reported in literature, ${ }^{[5]}$ and most publications were outside sub-Saharan Africa. Few evidences of optimal treatment strategy exist. Surgical excision of tumours remains the most accepted treatment modality. ${ }^{[6]}$ Other treatments modality include chemotherapy and radiotherapy. Osteosarcomas of the skull are highly aggressive tumours and have a guarded prognosis. ${ }^{[7]}$

This report aimed to describe the profile of two uncommon cases of osteosarcoma of the skull managed in our institution.

\section{Case presentation}

\section{Case one}

A 48-year-old woman was admitted to our neurosurgical centre with complaint of a rapidly progressing forehead swelling of eight-month duration (figure 1). There is associated headache and change in personality (irrational behaviour and lack self-care) but no seizure or altered conscious level. Physical examination revealed a mass on the frontal area, bony-hard, non-tender, measuring about $20 \times 32 \mathrm{~cm}$, not attached to overlying skin but fixed to underlying structures (figure 1). Glasgow coma score was 14/15 (deficit-V4) and both pupils were $3 \mathrm{~mm}$ sluggishly reacted to light. No cranial nerve deficits noted. No motor or sensory deficits. Examination of other systems showed no abnormality of note. Brain computerised tomography scan revealed a huge mixed density frontal bone mass with osteolytic and osteoblastic components, and both intracranial (extra-axial) and extracranial extensions (figure $1 \& 2$ ). Full blood counts showed anaemia $(\mathrm{Hb}-8.8 \mathrm{~g} / \mathrm{dl})$ which was corrected and other blood tests were normal.

She subsequently had gross total excision of frontal mass with about $1 \mathrm{~cm}$ normal bone margin via a bicoronal approach (figure 4) and cranioplasty with methylmethaacrylate. Histopathology confirmed osteosarcoma (figure 5). Post-operative outcome was good (no adverse events) and was referred to radio-oncologist for radiotherapy which she has not had due to logistics reasons. The patient remained in good condition for about three months before a sudden

deterioration in clinical state and death at peripheral hospital close to her domain.

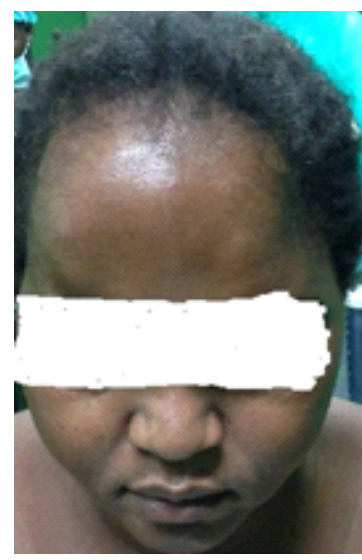

Figure 1: bi-frontal swelling.

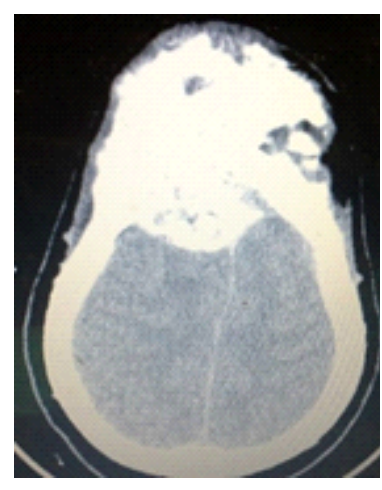

Figure 3: Axial cut CT scan (Tissue window).

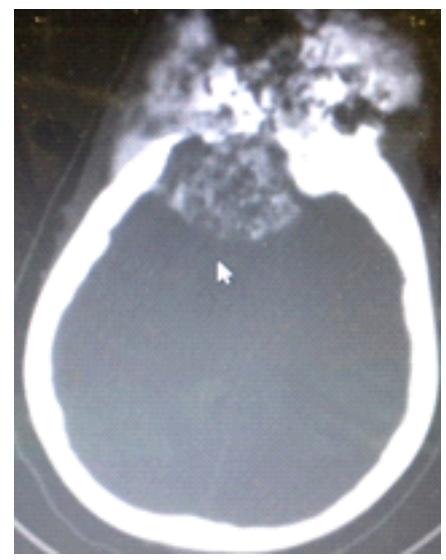

Figure 2: Axial cut CT scan (bone window).

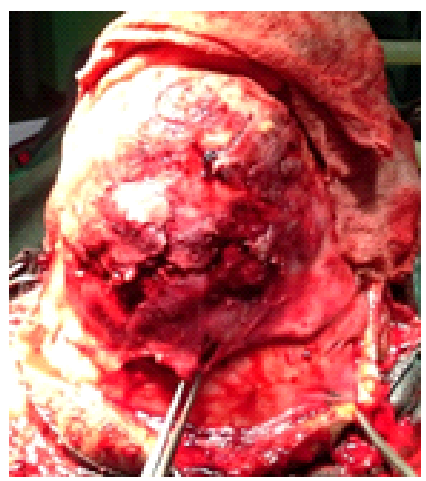

Figure 4: Intraoperative photograph showing tumour.

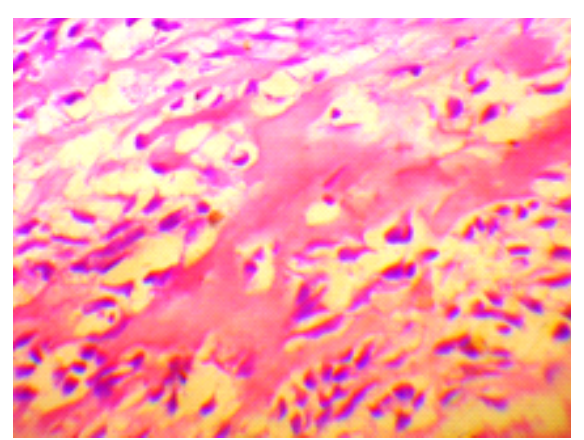

Figure 5: Photomicrograph. 


\section{Case two}

The second case was a 2 year old child who presented with rapidly progressing skull swelling noticed six months before presentation. There was associated weight loss, refusal to feeds and occasional crying. On physical examination, he was conscious, cachectic and pale. Glasgow coma score was 15 , pupils were $3 \mathrm{~mm}$ and reacted normally to light, no obvious cranial nerve palsy. There was bony hard frontoparieto-occipital mass with distended scalp veins, both anterior and posterior fontanelles were close. Cranial computerised tomography scan showed a mixed density skull mass with both osteolytic and osteoblastic components. The child was optimised and had subtotal excision of the skull tumour (figure $6 \& 7$ ) and died on table from profuse uncontrollable bleeding from residual bone tumour edges. Histopathology confirmed osteosarcoma.

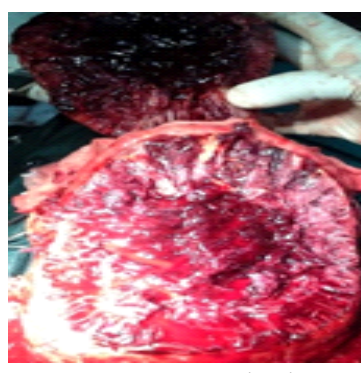

Figure 6: Intraoperative image.

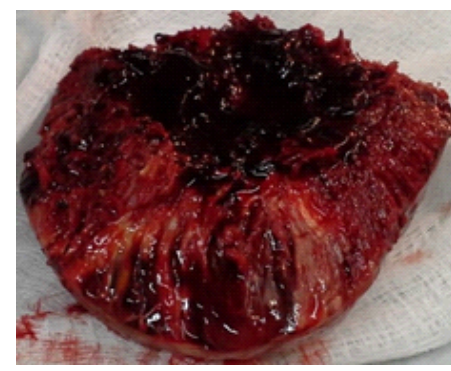

Figure 7: clinical photograph after subtotal excision.

\section{Discussion}

Osteosarcoma involving the skull is uncommon and mostly occurs in young individuals between $1^{\text {st }}$ to $3^{\text {rd }}$ decade of life. ${ }^{[]}$ Its commonly seen in males more than the females. ${ }^{[8-10]}$ In contrasts, to our index cases that were both females. The most common presenting clinical feature is focal swelling with or without pain. ${ }^{[7,11]}$ This is comparable to our patients that had a localised cranial vault swelling associated with pain. Computerised tomography scan is a useful imaging modality in assessing characteristics of the lesion, extents of the tumour, state of the surrounding brain as well as guiding surgical planning. The present cases had cranial computerised tomography scan which shows mixed density lesion with both osteolytic and osteoblastic components. Computerised tomography (CT) scan of the chest can be used to assess the presence of lung, pleura and chest wall metastasis in patients with osteosarcoma. ${ }^{[12]}$ Our patients did not have any clinical or radiological (chest radiograph) features of pulmonary metastasis, as such, chest CT scan was not done. Radionuclide bone scan is an important tool used in assessment of skeletal spread of osteosarcoma and other malignant conditions. ${ }^{[6,12]}$ Our patients could not have radionuclide bone scan because of unavailability and cost of bone scan. Usually, magnetic resonance imaging (MRI) is not commonly indicated in evaluation of patients with osteosarcoma. ${ }^{[13]}$ However, Contrast enhanced MRI can be used for evaluating soft tissue involvement such as: brain parenchyma, dura mater, cerebral arteries or sinuses, bone marrow, tumors involving the skull base ${ }^{[5,13]}$ The differential diagnosis of osteosarcoma of the cranial vault includes fibrous dysplasia, osteochondroma and chondrosarcoma. The definite differentiation of the aforementioned differentials cannot be made with certainty without histopathological examinations.
The exact cause of osteosarcoma is unknown, but notable risks factors for development of cranial osteosarcoma include antecedent irradiation or chemotherapy, Paget disease, fibrous dysplasia, trauma and chronic osteomyelitis. ${ }^{[9]}$ No risk factor was identified in our patients. Osteosarcomas have been classified into different subtypes based on histological characteristics: chondroblastic, osteoblastic, fibroblastic, telangiectatic, periosteal, small cell and parosteal osteosarcomas. ${ }^{[14]}$ Osteosarcoma was graded into low, intermediate or high grade, based on the extent of cellular atypia and architectural distortion. ${ }^{[15]}$ Our cases had high grade tumor. The most accepted treatment options for patient with cranial osteosarcoma is complete surgical excision followed by chemo-radiation in selected cases. Many chemotherapeutic and radiation regimens were documented in the literature but the most popular chemoradiation modality is administering six cycles of doxorubicin, cisplatin, methotrexate and ifosfamide followed by a fractionated dose of 50 Gray of radiotherapy. ${ }^{[5]}$ Our first case, had surgical excision and was referred to radio-oncologists for chemoradiation. There is possibility of dural and brain involvement and recurrence in patients with osteosarcoma of the skull. Our patients had only dural involvement warranting its excision but no brain invasion observed. Recurrence could occur in osteosarcomas and found to be reduced significantly when involved dura is resected with the tumor completely, ${ }^{[6]}$ but our cases had excision of the affected dura and did not survive long enough to develop recurrence.

\section{Conclusion}

The rarity of osteosarcoma of the skull leads to paucity of standardise treatment algorithm, despite poor prognosis. We recommend total surgical excision of the tumour and cranioplasty at same sitting especially in our environment where radiotherapy services are not always available for use and patients have to settle hospital bills from their pocket.

\section{References}

1. Oda D, Bavisotto LM, Schmidt RA, et al. Head and neck osteosarcoma at the University of Washington. Head Neck 1997;19:513-23.

2. Bouali S, Bouhoula A, Boubaker A, Ben Said I, Kallel J, Aouij L et al. A case Report of Osteosarcoma of the Skull. Journal of Modern Neurosurgery, 2014, 4, 105-109

3. Bose, B. (2002) Primary Osteogenic Sarcoma of the Skull. Surgical Neurology, 58, 234-240.

4. Vasquez L, Tejada V, Maza I and Mendoza R. Primary osteosarcoma of the skull in teenager. BMJ Case Reports CP 2019;12:e 229585.

5. Guangyong W, Qi L, Yu L. Primary osteosarcoma of frontal bone. A case report and review of literature. Medicine (2017) 96:51(e9392)

6. Federman N, Bernthal N, Eilber FC, et al. The multidisciplinary management of osteosarcoma. Curr Treat Options Oncol 2009;10: 82-93.

7. Haque F, Fazal ST, Ahmad SA, Abbas SZ and Naseem S Primary osteosarcoma of the skull. Australasian Radiology (2006) 50, 63-65

8. Fernandes GL, Natal MRC, Cruz CLP, Nascif RL, Tsuno NSG, Tsuno MY. Primary osteosarcoma of the cranial vault. Radiol Bras. 2017 Jul/Ago;50(4): 263-265

9. Gupta S, Chitra S, Singh D. Primary Osteogenic Sarcoma of Skull Bone-A rare clinical presentation. The Internet Journal of Oncology. 2008 Volume 6 Number 2.

10. Mascarenhas L, Peteiro A, Ribeiro CA, Magalhaes Z, Romao H, Magalhaes F et al. Skull osteosarcoma: illustrated review. Acta Neurochir (Wien). 2004; 146:1 235-9.

11. K. Gangadhar K, Santhosh D. Primary Skull Osteosarcoma: MDCT valuation and Histopathological Correlation in Two Cases. The Neuroradiology Journal 25: $188-192,2012$

12. Mascarenhas L, Peteiro A, Ribeiro CA, et al. Skull osteosarcoma: illustrated review. Acta Neurochir (Wien) 2004;146:1235-9.

13. Laine FJ, Nadel L, Braun IF: CT and MR imaging of the central skull base. Part 2. Pathologic spectrum. Radiographics 10: 797-821, 1990

14. Gadwal SR, Gannon FH, Fanburg-Smith JC, et al. Primary osteosarcoma of the head and neck in pediatric patients: a clinicopathologic study of 22 cases with a review of the literature. Cancer 2001;91:598-605.

15. MathkourM, Garces J, Beard B, et al. Primary high-grade osteosarcoma of the clivus: a case report and literature review. World Neurosurg 2016; 89: 730.e9-13. 